\title{
Sequence and Spread Spectrum Time Domain Reflectometry for Transmission Line Analysis
}

\author{
Jacklyn Reis ${ }^{a}$, Agostinho L.S. Castro ${ }^{b}$, João C.W.A Costa ${ }^{a}$, Jaume R.I. Riu ${ }^{c}$, and Klas \\ Ericson ${ }^{c}$ \\ ${ }^{a}$ Applied Electromagnetism Laboratory, Federal University of Pará, DEEC, Belém-PA, Brazil; \\ ${ }^{b}$ Signal Processing Laboratory, Federal University of Pará, DEEC, Belém-PA, Brazil; \\ ${ }^{c}$ Ericsson, Kista, Stockholm, Sweden
}

\begin{abstract}
This paper describes Sequence Time Domain Reflectometry (STDR) and Spread Spectrum Time Domain Reflectometry (SSTDR), which utilizes concepts from direct sequence spread spectrum communications, as a technique for detecting impedance mismatches in telephone lines (twisted pair). The aim of this paper is to present methodologies for characterizing a subscriber loop, which is used for Digital Subscriber Line (DSL) technology, based on STDR and SSTDR tests. Those tests enable the TDR functionality to be incorporated into a DSL transceiver integrated circuit eliminating the need for costly test equipment. In addition to the cost savings, the characteristics of the STDR and SSTDR offer improved spectral compatibility, interference immunity and fault resolvability.
\end{abstract}

Keywords: Loop qualification, digital subscriber line, sequence time domain reflectometry, spread spectrum

\section{INTRODUCTION}

Reflectometry methods have been used for locating faults (impedance mismatches) on wires for decades. These methods send a high frequency signal down the line, which reflects back at impedance mismatches such as open or short circuits, gauge changes and specially in bridged taps. ${ }^{1}$ The difference (time delay) between the incident and reflected signal is used to locate the impedance discontinuity on the wire. The nature of the input signal is used to classify each type of reflectometry test. Time Domain Reflectometry (TDR) uses a fast rise time pulse. ${ }^{1-3}$ Frequency Domain Reflectometry (FDR) including Phase Detection Reflectometry (PD-FDR), and Mixed Signal Reflectometry (MSR), uses sine-wave signals to locate the fault on the wire. Multicarrier Reflectometry (MCR) uses a combination of sine waves with random phases. ${ }^{4}$ Sequence Time Domain Reflectometry (STDR) uses a pseudo-noise sequence (PN sequence), and Spread Spectrum Time Domain Reflectometry (SSTDR) uses a sine-wave-modulated $\mathrm{PN}$ sequence. ${ }^{4,5}$

Some traditional reflectometry methods should not be applied under specific conditions, e.g. very short loops $(\leq 200 \mathrm{~m})$ or noisy environment. Besides, those techniques may not detect the impedance discontinuity or detect it with low accuracy. Additionally, it has been shown that traditional reflectometry methods are not effective for location of small fault (which generates weak reflections such as gauge change or far discontinuity) on telephony lines for instance ${ }^{1}$ the reason for that is the returned reflections are smaller than the reflections that are generated from others impedance discontinuities in the line such as bridged taps, open and short circuits. Besides, the subscriber telephone loop exhibits distributed RLC behavior which causes the slowly decaying in the test signal. If the reflection is very strong, this behavior may be neglected, whereas in the case of weak reflections, this approximation turns out to be too harsh. ${ }^{1}$ Another difficulty in detecting weak reflection lies on the fact that there are spurious reflections and/or noise which interferes in the reflection signal of interest (used for locating faults); then making difficult the location of the discontinuity. It is worth emphasizing that the shorter is the telephony loop, the higher frequency should be used in the test pulse to provide high accurate result for wiring fault location.

Further author information:

J. Reis, A.L.S Castro, J.C.W.A. Costa: E-mail: jacklyn, agcastro, jweyl@ufpa.br

J.R.I. Riu, K. Ericsson: E-mail: jaume.rius.i.riu, klas.ericson@ericsson.com 
The main goal of this paper is to provide better results with high accuracy, for fault location in the subscriber telephone loop than the ones provided by traditional reflectometry techniques by using sequence and spread spectrum TDR. Gauge changes, bridged taps as well as total loop length are the main variables that will be analyzed in this paper.

The present paper is organized as follows. The discussion about reflectometry methods including traditional, sequence, and spread spectrum TDR is analyzed in Section 2. The details about the implementation of STDR and SSTDR techniques are outlined in Section 3. Section 4 presents the results obtained from computational simulations. Section 5 presents the results based on measured data in real cables. Finally, the conclusions and next works are addressed in Section 6.

\section{BACKGROUND}

This section discusses the theoretical concepts about traditional TDR and S/SSTDR. Traditional TDR generally uses a rectangular pulse (high-peak power pulse) with high-frequency band as a test signal to be transmitted along the loop, and to perform such a test the line should be inactive (without transmission or interfering signal).

The transmission characteristics of the TDR pulse have several inherent drawbacks. A high-peak power pulse is likely to be a significant disturber to other pairs in the binder. This type of crosstalk will most certainly violate spectral compatibility standards in DSL transmission for instance. ${ }^{6}$ A second drawback is the requirement to produce pulses at a variety of different levels and with a variety of different durations. This equates to a requirement on the test equipment to transmit signals and to receive signals with a large dynamic range. This alone makes the possibility of incorporating this type of signal into a DSL transceiver integrated circuit very questionable. $^{7}$

On the other hand, S/SSTDR uses a PN sequence (base band or pass band) as a test signal and by utilizing concepts of Direct Sequence Spread Spectrum (DS-SS) communications, ${ }^{8-10}$ a TDR capability has been developed which may be efficiently and cost effectively integrated into a DSL transceiver for instance.

\subsection{Traditional TDR}

TDR is widely used in the deployment of DSL services. When the line is provisioned, it is one of several test measurements used to ascertain the characteristics, topology and ultimately the capacity of the line. A measurement or reflectogram is obtained by connecting the test equipment to the line and bursting a high power pulse onto the line and measuring the reflections (echoes). Those reflections results from impedance mismatches on the line. The amount of the incident signal that is reflected toward the transmitter is determined by the reflection coefficient $\rho$ given by

$$
\rho=\frac{Z_{2}-Z_{1}}{Z_{2}+Z_{1}}
$$

where $Z_{1}$ is the impedance looking into the line from the transmitter and $Z_{2}$ is the impedance looking into the anomaly. By analyzing the position and shape of the echoes, a number of characteristics about the line can be determined including the distance to opens/shorts, the location and length of bridged taps or gauge changes, as well as the total loop length. ${ }^{1}$

The distance to the fault is computed according to

$$
d=\frac{T_{p} \cdot V O P}{2}
$$

where $T_{p}$ is the round trip propagation time to the fault and back and $V O P$ is the velocity of propagation on the twisted pair. This term is typically about two-thirds the speed of light, but, the exact value depends on the physical wire.

Using this basic methodology, other loop attributes can be detected and analyzed. For example, a bridged tap terminated with an open will exhibit a first echo resembling a short and a second echo resembling an open. 
The first echo has negative polarity because the bridged tap is in parallel with the remaining portion of the line. The combined impedance is less than that of the line without the bridged tap and so the reflection coefficient is negative. A positive echo is reflected from the open at the end of the bridged tap. The length of the bridged tap can be found by computing the distance between the negative and positive echoes. ${ }^{1}$

Implicit in (2) is the test ability of equipment to estimate the propagation time $T_{p}$. The estimation performance is limited by interference coupled into the line from adjacent pairs in the same binder and by the dispersive nature of the line. As the distance to faults increases, both the attenuation and distortion to the pulse increases. Therefore, typical TDRs have the capability to adjust signal parameters to aid in the search for faults. The two parameters available for adjustment are the transmit level of the pulse and the duration of the pulse. A narrow pulse is generally preferred since this directly impacts the resolvability of closely spaced faults. But, this is only effective at short distances. At larger distances, increasing the signal level and pulse duration is needed to produce sufficient energy for good detectability. Also, the types of disturbers present in the binder may necessitate modifying the transmit signal. ${ }^{3}$

\subsection{Sequence and Spread Spectrum TDR}

Some concepts on spread spectrum $\mathrm{TDR}^{5,11,12}$ as well as sequence $\mathrm{TDR}^{4,7}$ are discussed now. Those two reflectometry techniques use spread spectrum modulation to transmit a test signal $s(t)$ (PN sequence) over the line. Besides spread spectrum modulation, SSTDR also uses PSK (Phase Shift Keying) modulation to create the transmitted signal. On the other hand, STDR uses only the PN signal as the transmitted signal. A schematic setup for STDR and SSTDR circuits is depicted in Figure 1.

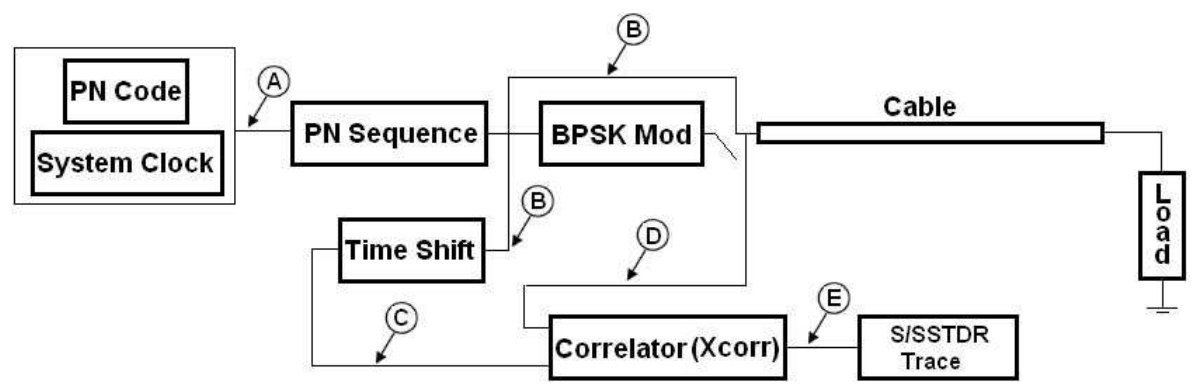

Figure 1. S/SSTDR schematic setup.

The schematic for S/SSTDR implementation shown in Figure 1 creates first a specific kind of PN code (length, frequency, amplitude, etc), depending on the nature of the test, depicted in point $A$ at Figure. This code may be a M-Sequence Code (Maximal Length Sequence), a Gold Code, or a pair of complementary codes such as Golay Codes. ${ }^{8-10,13,14}$ Next, this signal is injected onto the PN Sequence block to generate the waveform of PN sequence $s(t)$ indicated in point $B$. This signal $s(t)$ is transmitted along the cable in case of sequence TDR. In the case of SSTDR, the PN sequence passes through the BPSK block, thus it performs the BPSK (Binary PSK) modulation of the PN sequence creating the DS (Direct Sequence) Spread Spectrum Signal. ${ }^{9,10}$ The total signal from the loop named by $s^{\prime}(t)$ (it may be obtained from STDR or SSTDR tests) in point $D$, including any noise signals and reflections from impedance discontinuities in the cable, is fed into the Correlator block along with a delayed copy of the transmitted signal $s(t+\tau)$. This delayed signal $s(t+\tau)$ (point $C$ ) is obtained in Time Shift block by adding a number of zeros in the signal $s(t)$. In the Correlator block, the cross-correlation between the received signal $s^{\prime}(t)$ (point $D$ ) and the delayed signal $s(t+\tau)$ (point $C$ ) is performed (point $E$ ). Mathematically, this cross-correlation is defined as it follows

$$
R_{s s^{\prime}}(\tau)=\frac{1}{K T_{c}} \int_{0}^{K T_{c}} s(t+\tau) s^{\prime}(t) d \tau
$$

where $T_{c}$ is the chip duration of the PN sequence, $K$ is the length of the PN sequence, and $K T_{c}$ is the PN sequence duration in seconds. Thus, the last step is to plot the S/SSTDR trace in time domain; S/SSTDR Trace block carries out that curve. 
The location of the various peaks in the full correlation $R_{s s^{\prime}}(\tau)$, defined in $(3)$, indicates the location of impedance discontinuities such as open circuits, short circuits, bridged taps and gauge changes along the loop.

Spread spectrum signals, both in baseband (STDR) and modulated (SSTDR), are detectable through crosscorrelation, regardless the presence of noise, other digital signals and reflections on the line. ${ }^{5,11,12}$ The ability to pick out the signal is due to processing gain $L_{c}$ defined as it follows

$$
L_{c}=\frac{T_{b}}{T_{c}}=\frac{R_{c}}{R_{b}}=\frac{W}{R_{b}}
$$

where $W$ is the bandwidth of the spread-spectrum signal, $T_{b}$ denotes the duration of one entire $\mathrm{S} / \mathrm{SSTDR}$ sequence (considering the entire sequence equal to one bit in communication-system terms), $R_{c}$ represents the chip rate of the $\mathrm{PN}$ sequence in chips per second, and $R_{b}$ is the bit rate, which in this case is the number of full sequences per second. ${ }^{11}$

\section{S/SSTDR IMPLEMENTATION}

This section describes algorithm implementation for performing sequence and spread spectrum TDR in telephone loops used for xDSL transmission. First, methods for generating PN sequences are discussed; Golay codes (complementary codes) and Maximum-Length codes (M-sequences) are briefly outlined. Next, the methods for performing correlation, auto-correlation and cross-correlation, are described.

\subsection{PN Sequences}

Depending on the application, the PN sequences may be a crucial factor for limiting or not the method. To define which $\mathrm{PN}$ sequence is the best for a specific application, many variables can be taken into account. Correlation, code implementation, hardware implementation and time processing are some of them. We are focused in discussing correlation properties and code implementation of the PN sequences.

The basic idea of S/SSTDR is to use a signal with a sufficiently narrow auto-correlation function, similar to the Dyrac's delta, rather than a short duration signal itself (high-frequency pulse); the response resolution of the $\mathrm{S} / \mathrm{SSTDR}$ is determined by the width of the auto-correlation function of a probe signal which may be long and with a large duty cycle. ${ }^{14}$

The optimal PN sequence depends on the nature of the application. In our application, the best PN sequence should have a narrow main lobe with a high peak and to present the lowest side lobes in its auto-correlation. It is worth emphasizing that such a PN sequence is restricted for applications where only one PN sequence will be used at a time, that is the test is performed in only one cable; without adjacent interfering lines. ${ }^{11}$ To obtain such a PN sequence with a single auto-correlation peak and the lack of side lobes, we decided to use a pair of complementary sequences (Golay codes). Adding these two complementary sequences, an auto-correlation function is obtained without side lobes. ${ }^{13,14}$ Figure 2.a shows the waveforms of a pair of complementary PN sequences of length $K=32, P N$ Sequence $A$ and $P N$ Sequence $B$, transmitted into the line. Also, Figure 2.b shows the same complementary signals but in pass band used in SSTDR. Figure 3.a and 3.b show the auto-correlation functions for STDR and SSTDR tests. The correlations of interest identified by Auto-correlation STDR and Auto-correlation STDR are obtained by adding auto-correlation of $P N$ Sequence $A$ to the auto-correlation of $P N$ Sequence $B ;^{13,14}$ this sum allows to obtain an auto-correlation without side lobes as depicted in Figure 3.

\subsection{Correlation}

S/SSTDR may be performed by using two kinds of test signals. One of them utilizes a high-peak auto-correlation as a test signal to be injected into the line. The auto-correlation function may be defined as it follows in equation (3); however, the correlation is carried out by using $s(t)$ only instead of $s^{\prime}(t)$ and $s(t)$.

In that case, the PN sequence is used only for obtaining the auto-correlation with desired characteristics. This method is very simple to be code implemented, on the other hand, the hardware implementation limits its application for telephone lines carrying xDSL signals. The reason for such a limitation comes from the fact that is too difficult to generate that periodic high-frequency signal. 

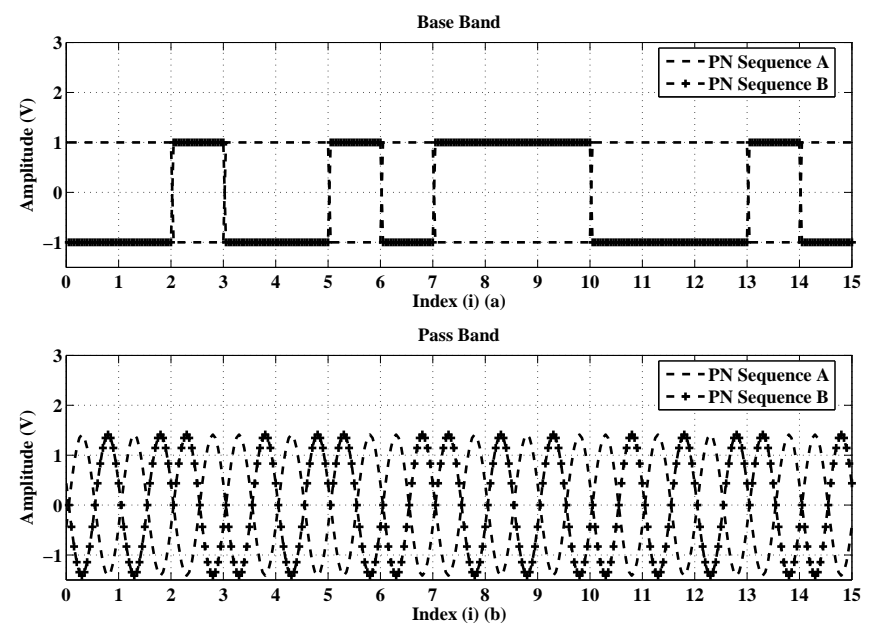

Figure 2. (a) Waveforms (A and B) for STDR. (b) Waveforms (A and B) for SSTDR.
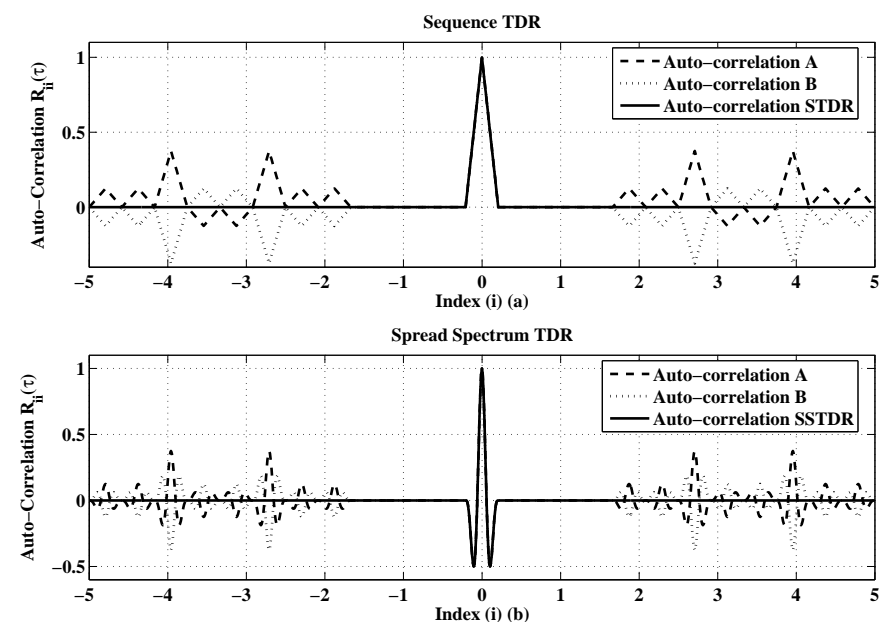

Figure 3. (a) Auto-correlation function of complementary codes A and B for STDR. (b) Auto-correlation function of complementary codes A and B for SSTDR.

The second method for performing sequence TDR is to utilize PN sequence as a test signal to be transmitted down the cable. After transmission, the received PN signal is correlated with a delayed copy of transmitted PN signal, thus the correlator output may be analyzed for locating impedance mismatches in the cable. Those two methods are outlined next.

\subsubsection{Auto-Correlation Method (ACM)}

This method for performing S/SSTDR utilizes an auto-correlation function as a test signal as described before. This signal is transmitted into the line, thus the TDR trace may be obtained by convolution of echo impulse response $h(t)$ of the line and the auto-correlation function of the PN sequence as shown below

$$
\begin{aligned}
\operatorname{STDR}_{A C M}(t) & =s(t) \oplus s^{\prime}(t) \\
& =s(t) \oplus[s(t) \otimes h(t)] \\
& =[s(t) \oplus s(t)] \otimes h(t)
\end{aligned}
$$


where $\oplus$ denotes correlation function, $\otimes$ denotes convolution in time domain, $s(t)$ is the PN sequence transmitted into the line, $h(t)$ is the echo impulse response of the line, and $s^{\prime}(t)$ is the received signal from the line which is equal to the convolution between $h(t)$ and $s(t)$.

Using associative property of convolution, then it enables to calculate the auto-correlation function of the PN sequence before transmitting it into the line. This procedure has the same effect of transmitting the autocorrelation function as a test signal into the line as defined in (5).

This method is quite simple to be code implemented, however it limits the implementation in a real xDSL transceiver. This implementation would require a high-clock system to generate the high-peak auto-correlation function as a test signal.

\subsubsection{Cross-Correlation Method (CCM)}

Although this method provides the same result of the method which utilizes the auto-correlation function as a test signal, this method of performing STDR is quite simple to be implemented in a real xDSL transceiver. This method requires less hardware complexity because transmitting a high-frequency digital data into the line is easier than transmitting a high-peak auto-correlation function.

The implementation consists, first, in generating a PN sequence with the characteristics discussed in preview sections. Then, such a PN sequence $s(t)$ is probed into the line by mean of convolution with echo impulse response $h(t)$; thus the PN sequence is the test signal. The reflected signal $s^{\prime}(t)$ from the line is equal to the convolution $s^{\prime}(t)=s(t) \otimes h(t)$ as discussed before. The difference between the two methods comes from the fact that the correlation, performed after transmission, in this case is a cross-correlation function. It is worth emphasizing that spread-spectrum technique is applied at the transmitter for obtaining the TDR trace; the received signal from the loop $s^{\prime}(t)=s(t) \otimes h(t)$ is then correlated with a delayed copy of the transmitted signal $s(t+\tau)$ as it follows in equation below.

$$
\begin{aligned}
\operatorname{STDR}_{C C M}(t) & =s(t+\tau) \oplus s^{\prime}(t) \\
& =s(t+\tau) \oplus[s(t) \otimes h(t)]
\end{aligned}
$$

\section{SIMULATED RESULTS}

This section discusses and presents the results based on computational simulation using Matlab. Basically, these results comprise performing S/SSTDR with and without noise (Additive White Gaussian Noise - AWGN) in three different telephone line topologies: the first topology consists in single loops (only one line segment) open circuit terminated, the second topology consists in loops presenting mixture of gauges $(0.40 \mathrm{~mm}$ and $0.50 \mathrm{~mm})$, the last topology consists in loops presenting one bridged tap (50m-length) with its location varying along the line (at the beginning, at the center, and at the end of the line).

Those simulations were carried out by using complementary PN sequences (Golay Codes A and B) with length $K=128$ (processing gain $L_{c}=128$ ) and frequency $f=30 M H z$. Additive noise (AWGN) was set with zero mean and variance $\sigma^{2}=10^{-4}$.

\subsection{Line Topology 1}

Table 1 shows the results obtained from computational simulation of S/SSTDR, using the test signal described before, as well as traditional TDR (Pulse Width $=100 \mathrm{~ns}$ ). In those simulations, both methods of S/STDR, $\mathrm{ACM}$ and CCM, provided the same results for calculating the total loop length. Also, Figure 4 shows the results obtained for a telephone line of $200 \mathrm{~m}(0.40 \mathrm{~mm})$. In this case, traditional TDR was not capable to calculate the total loop length. 


\subsection{Test Loop 2}

Table 2 summarizes the results for S/SSTDR and traditional TDR (Pulse Width = 100ns) applied to telephone loops with two kinds of gauge $(0.40 \mathrm{~mm}$ and $0.50 \mathrm{~mm}$ ). Also, Figure 5 (loop composed by two serial segments, first $50 \mathrm{~m}$-length with $0.50 \mathrm{~mm}$ gauge, and second $150 \mathrm{~m}$-length with $0.40 \mathrm{~mm}$ ) presents the TDR traces comparing $\mathrm{S} / \mathrm{SSTDR}$ to traditional TDR. According to those results, it is clear that S/SSTDR is more accurate and immunity to the white noise for calculate the total loop length. However, it is important emphasizing that both methods fail to detect the weak reflections caused by gauge changes.

\subsection{Test Loop 3}

Table 3 summarizes the results for S/SSTDR and traditional TDR (Pulse Width $=100 \mathrm{~ns}$ ) applied to 200mtelephone loops with one bridged tap. Also, Figure 6 (200m-length loop and one 50m-length bridged tap positioned at $50 \mathrm{~m}$ from the source) presents the TDR traces comparing S/SSTDR to traditional TDR. It is important to mention that both the bridged-tap and the line is formed by the same gauge cable, $0.40 \mathrm{~mm}$.

Table 1. Results for line topologies 1.

\begin{tabular}{|c|c|c|c|}
\hline Gauge $(\mathrm{mm}) /$ Length $(\mathrm{m})$ & TDR $(\mathrm{m})$ & STDR $(\mathrm{m})$ & SSTDR $(\mathrm{m})$ \\
\hline $0.40 / 200$ & - & 203.3 & 198.3 \\
\hline $0.40 / 100$ & 104.1 & 102.5 & 100.9 \\
\hline $0.50 / 200$ & - & 201.7 & 196.9 \\
\hline $0.50 / 100$ & 104.1 & 102.5 & 100.9 \\
\hline
\end{tabular}

Table 2. Results for line topologies 2 .

\begin{tabular}{|c|c|c|c|c|c|c|}
\hline S1 + S2 (Gauge/Length) & TDR & STDR & SSTDR & TDR & STDR & SSTDR \\
\hline $0.40 \mathrm{~mm} / 050 \mathrm{~m}+0.50 \mathrm{~mm} / 150 \mathrm{~m}$ & - & - & - & - & $201.7 \mathrm{~m}$ & $198.5 \mathrm{~m}$ \\
\hline $0.40 \mathrm{~mm} / 100 \mathrm{~m}+0.50 \mathrm{~mm} / 100 \mathrm{~m}$ & - & - & - & - & $203.3 \mathrm{~m}$ & $198.5 \mathrm{~m}$ \\
\hline $0.40 \mathrm{~mm} / 150 \mathrm{~m}+0.50 \mathrm{~mm} / 050 \mathrm{~m}$ & - & - & - & $216 \mathrm{~m}$ & $203.3 \mathrm{~m}$ & $198.5 \mathrm{~m}$ \\
\hline $0.50 \mathrm{~mm} / 050 \mathrm{~m}+0.40 \mathrm{~mm} / 150 \mathrm{~m}$ & - & - & 50.82 & $198.5 \mathrm{~m}$ & $201.7 \mathrm{~m}$ & $198.5 \mathrm{~m}$ \\
\hline $0.50 \mathrm{~mm} / 100 \mathrm{~m}+0.40 \mathrm{~mm} / 100 \mathrm{~m}$ & - & - & - & $206.5 \mathrm{~m}$ & $201.7 \mathrm{~m}$ & $198.5 \mathrm{~m}$ \\
\hline $0.50 \mathrm{~mm} / 150 \mathrm{~m}+0.40 \mathrm{~mm} / 050 \mathrm{~m}$ & - & - & - & - & $201.7 \mathrm{~m}$ & $196.9 \mathrm{~m}$ \\
\hline
\end{tabular}

Table 3. Results for line topologies 3.

\begin{tabular}{|c|c|c|c|}
\hline Test & S1 (calculated/real) & BT (calculated/real) & Total Length (calculated/real) \\
\hline TDR & $51.41 / 50 \mathrm{~m}$ & $51.39 / 50 \mathrm{~m}$ & $-/ 200 \mathrm{~m}$ \\
\hline STDR & $49.23 / 50 \mathrm{~m}$ & $50.77 / 50 \mathrm{~m}$ & $204.9 / 200 \mathrm{~m}$ \\
\hline SSTDR & $49.23 / 50 \mathrm{~m}$ & $50.77 / 50 \mathrm{~m}$ & $198.5 / 200 \mathrm{~m}$ \\
\hline TDR & $-/ 100 \mathrm{~m}$ & $54.0 / 50 \mathrm{~m}$ & $-/ 200 \mathrm{~m}$ \\
\hline STDR & $100 / 100 \mathrm{~m}$ & $50.9 / 50 \mathrm{~m}$ & $204.8 / 200 \mathrm{~m}$ \\
\hline SSTDR & $100 / 100 \mathrm{~m}$ & $49.3 / 50 \mathrm{~m}$ & $200.0 / 200 \mathrm{~m}$ \\
\hline TDR & $-/ 150 \mathrm{~m}$ & $-/ 50 \mathrm{~m}$ & $-/ 200 \mathrm{~m}$ \\
\hline STDR & $150.9 / 150 \mathrm{~m}$ & $55.6 / 50 \mathrm{~m}$ & $206.5 / 200 \mathrm{~m}$ \\
\hline SSTDR & $152.5 / 150 \mathrm{~m}$ & $46.0 / 50 \mathrm{~m}$ & $198.5 / 200 \mathrm{~m}$ \\
\hline
\end{tabular}




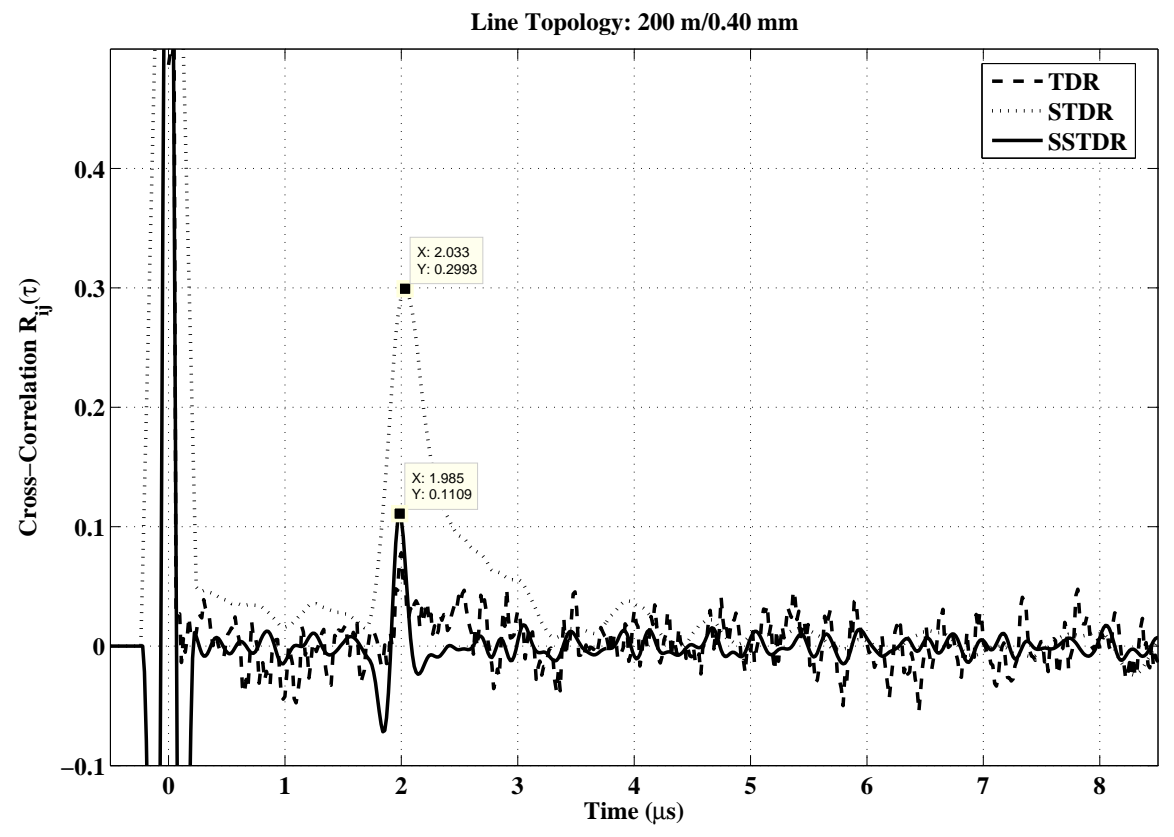

Figure 4. S/SSTDR versus traditional TDR for $200 \mathrm{~m} / 0.40 \mathrm{~mm}$.

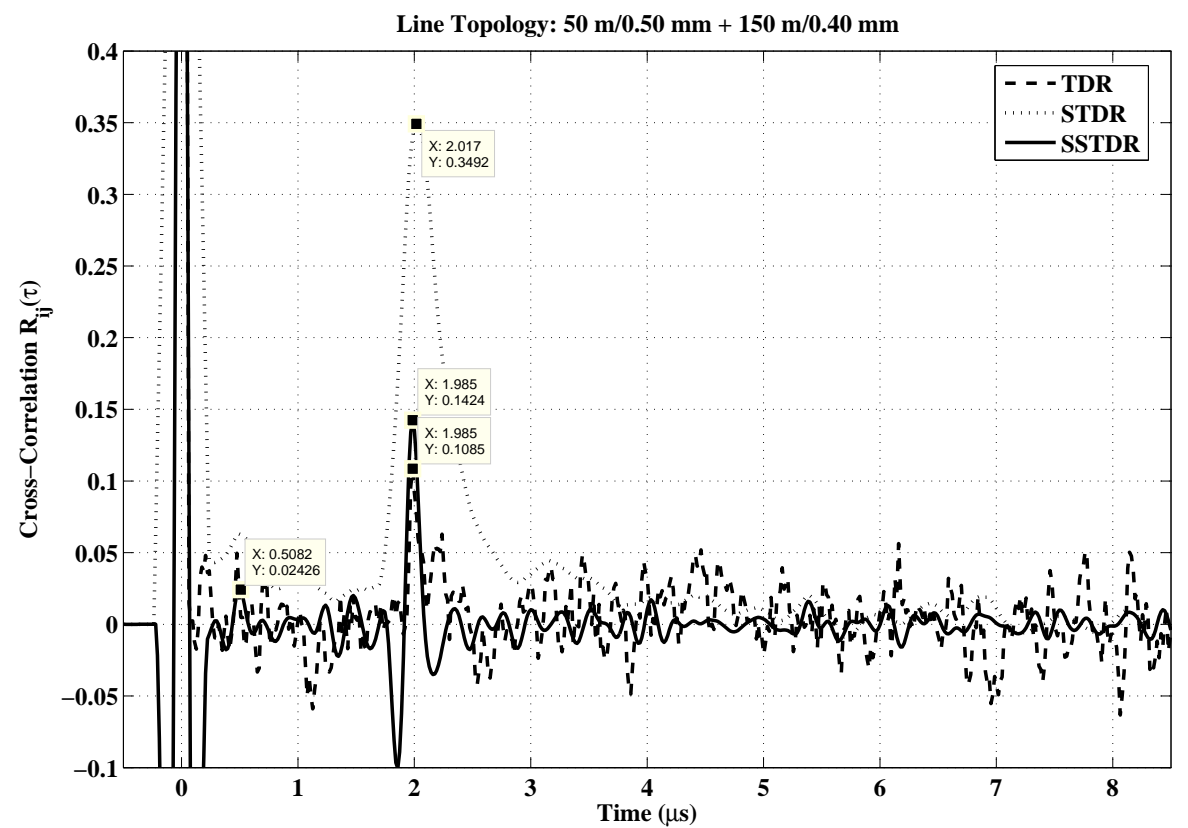

Figure 5. S/SSTDR versus traditional TDR for $50 \mathrm{~m} / 0.50 \mathrm{~mm}+150 \mathrm{~m} / 0.40 \mathrm{~mm}$. 


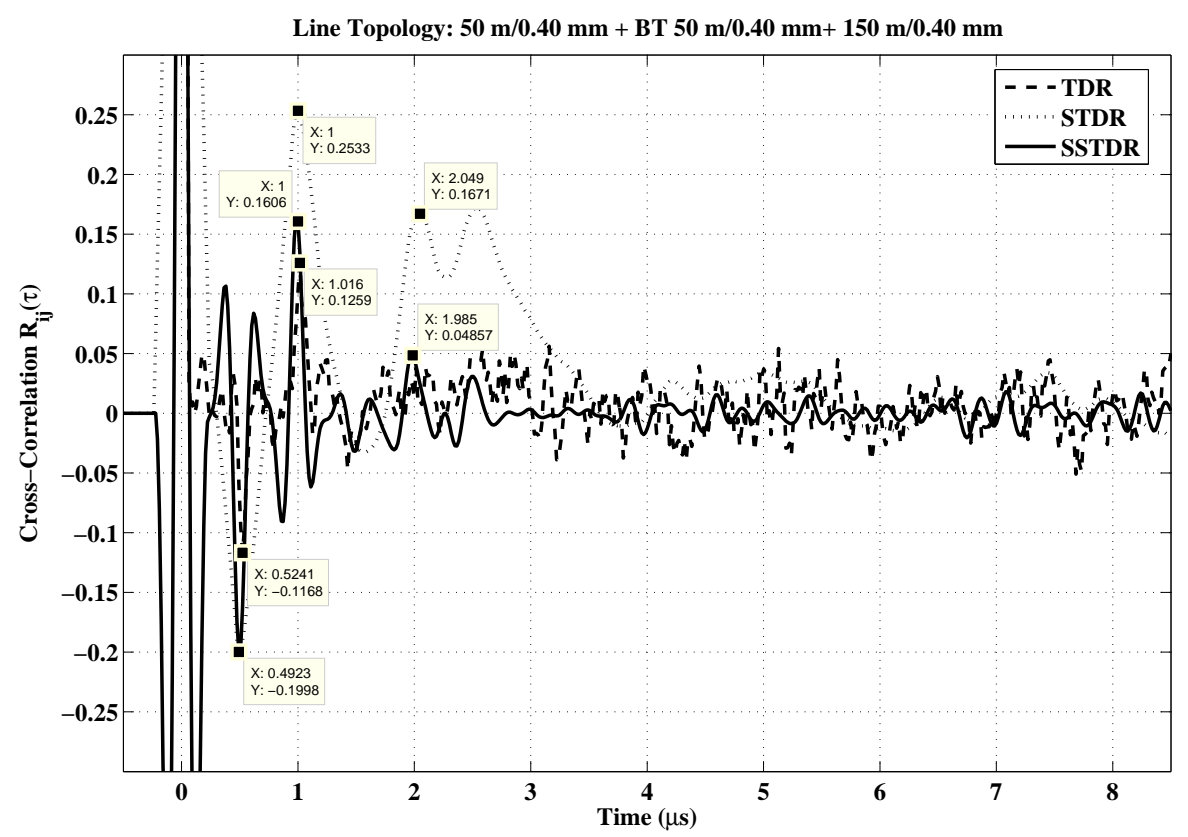

Figure 6. S/SSTDR versus traditional TDR for 0.40mm-loop with one bridged tap.

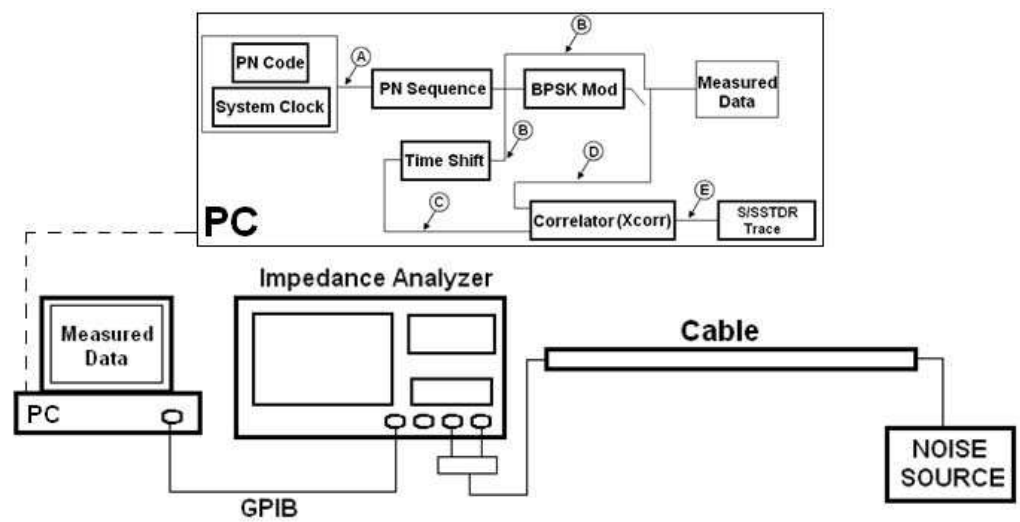

Figure 7. Schematic setup for performing S/SSTDR based on measured data.

\section{REAL CABLE TEST}

This section discusses a methodology for performing S/SSTDR in real cables. To guarantee that S/SSTDR may provide accurate results for calculate the total line length even in presence of noise, a measuring apparatus was implemented in lab to performing time domain reflectometry in real telephone lines.

Figure 7 shows the implemented apparatus. This apparatus first measures the input impedance of the line, in presence of white noise, in $30 \mathrm{MHz}$ frequency band through a high-accuracy impedance analyzer, ${ }^{15,16}$ then the measured data representing the the line is the input of the algorithm for performing S/SSTDR.

Figure 8 shows a result obtained for S/SSTDR compared to traditional TDR in real cables. As it can be seen, this result shows that S/SSTDR may provide better result for calculating the total line length even in presence of white noise. 


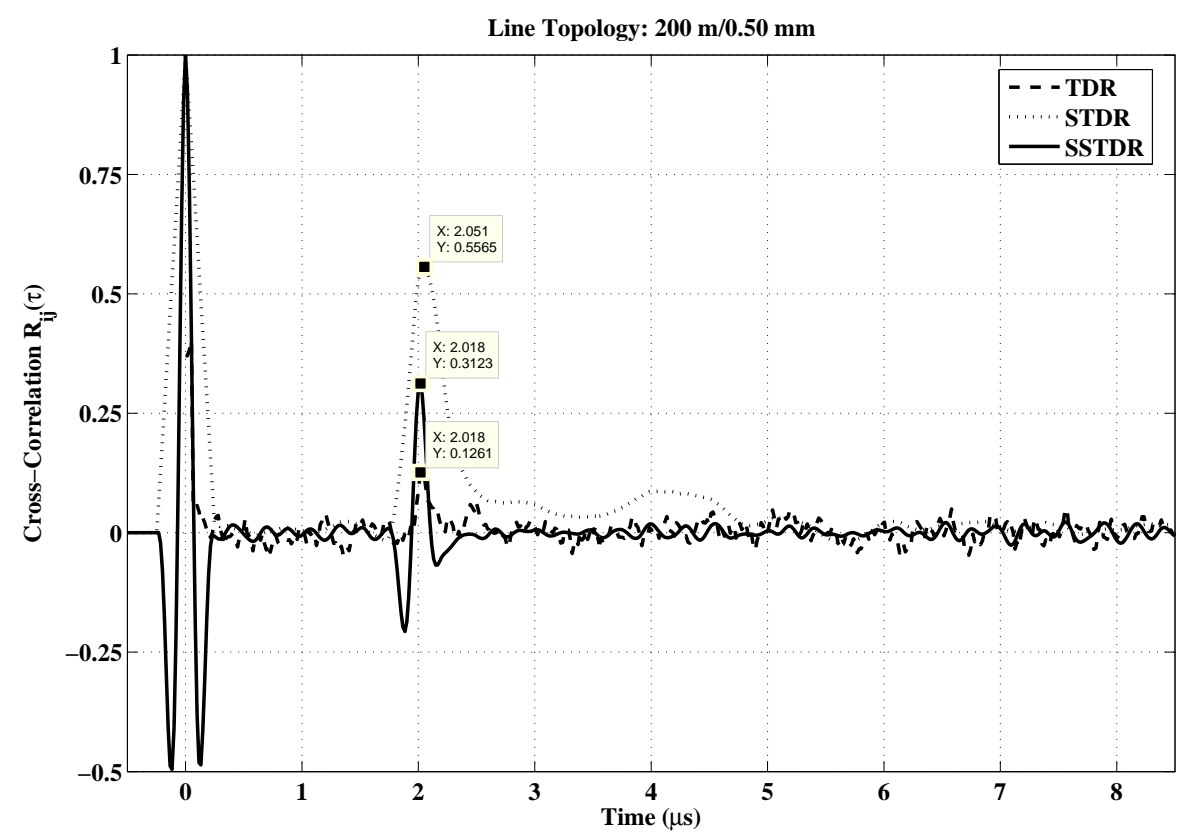

Figure 8. S/SSTDR versus traditional TDR for $200 \mathrm{~m} / 0.50 \mathrm{~mm}$-line. This result was obtained from a S/SSTDR test in real telephone cable.

\section{CONCLUSIONS}

This paper presented the usage of a new methods, sequence time domain reflectometry and spread spectrum time domain reflectometry, for performing reflectometry applied to analysis of transmission line, specially telephone lines. These methods shown to be feasible for performing efficiently reflectometry; they provide high accurate results when applied to very short loops $(\leq 200 \mathrm{~m})$, even though with mixture of gauges and bridged taps, compared to traditional TDR. The application of sequence and spread spectrum TDR also provides good results for calculation of total line length even in presence of white noise (AWGN). Additionally, sequence and spread spectrum TDR enables the implementation of reflectometry tests in DSL transceiver then no costly measuring equipment is required.

Next, this research will extend the application of sequence and spread spectrum TDR for longer loops in presence of noise and transmitting DSL signals (active loops).

\section{ACKNOWLEDGMENTS}

Some authors wish to acknowledge the support received from the Swedish Agency for Innovation Systems, VINNOVA, and the CELTIC Initiative within the EUREKA program, which partially enabled this work. 


\section{REFERENCES}

1. S. Galli and D.L. Waring, "Loop Make-up Identification via Single Ended Testing: Beyond Mere Loop Qualification," IEEE Journal on Selected Areas in Communications 20, pp. 923-935, June 2002.

2. S. Galli and K.J. Kerpez, "Single-Ended Loop Make-up Identification Part I: A method of analyzing TDR measurements," IEEE Transactions on Instrumentation and Measurements 55, pp. 538-549, April 2006.

3. K.J. Kerpez and S. Galli, "Single-Ended Loop Make-up Identification Part II: Improved Algorithms and Performance Results," IEEE Transactions on Instrumentation and Measurements 55, pp. 538-549, April 2006.

4. C.R. Sharma and C. Furse and R.R. Harrison, "Low-Power STDR CMOS Sensor for Locating Faults in Aging Aircraft Wiring," IEEE Sensors Journal 7, pp. 43-50, January 2007.

5. P. Smith, Spread Spectrum Time Domain Reflectometry. PhD thesis, Utah State University, 2003.

6. International Telecommunications Union - ITU, "Test Procedures for DSL Transceivers Standard - G.996.1," 1999.

7. K. Jones and G.I.S. Trevio and W.W. Jones and R.H. Jonsson, "Adaptive Method and Apparatus for Transmission Line Analysis," 2002.

8. S. Haykin, Communication Systems, Wiley, 4th ed., 1999.

9. John G. Proakis, Digital Communications, McGraw-Hill, 4th ed., 2001.

10. J.G. Proakis and M. Salehi, Communication System Engineering, Prentice-Hall, 2nd ed., 2002.

11. P. Smith and C. Furse and J. Gunther, "Analysis of Spread Spectrum Time Domain Reflectometry for Wire Fault Location," IEEE Sensors Journal 5, pp. 1469-1478, December 2005.

12. C. Furse, P. Smith, M. Safavi, and C. Lo, "Feasibility of Spread Spectrum Sensors for Location of Arcs on Live Wires," IEEE Sensors Journal 5, pp. 1445-1450, December 2005.

13. D.V. Sawarte and M.B. Pursley, "Crosscorrelation Properties of Pseudorandom and Related Sequences," Proceedings of The IEEE 68, pp. 593-619, May 1980.

14. F.J. Mendieta and A. Trevino and C.A. Martinez, "Complementary Sequence Correlations with Applications to Reflectometry Studies," Journal of The Mexican Society of Instrumentation 3, pp. 37-46, April 1996.

15. A. Technologies, "Impedance measurement handbook," 2003.

16. A. Technologies, "Adsl copper loop measurements," 2000. 